\title{
Life-Threatening Psoas Hematoma due to Retroperitoneal Hemorrhage in a COVID-19 Patient on Enoxaparin Treated With Arterial Embolization: A Case Report
}

\author{
Ishan Patel ${ }^{\mathrm{a}}$, Arda Akoluk ${ }^{\mathrm{a}}$, Steven Douedia, d, Vandan Upadhyaya ${ }^{\mathrm{a}}$, \\ Usman Mazahir ${ }^{\mathrm{b}}$, Eric Costanzo ${ }^{\mathrm{b}}$, Daniel Flynn ${ }^{\mathrm{c}}$
}

\begin{abstract}
Respiratory failure is presumptively caused by microvascular thrombosis in some patients with coronavirus disease 2019 (COVID-19) requiring therapeutic anticoagulation. Anticoagulation treatment may cause life-threatening bleeding complications such as retroperitoneal hemorrhage. To the best of our knowledge, we report first case of a COVID-19 patient treated with therapeutic anticoagulation resulting in psoas hematoma due to lumbar artery bleeding. A 69-year-old patient presented with fever, malaise and progressive shortness of breath to our hospital. He was diagnosed with COVID-19 by RT-PCR. Due to an abnormal coagulation profile, the patient was started on enoxaparin. Over the course of hospitalization, the patient was found to have hypotension with worsening hemoglobin levels. Computed tomography scan of the abdomen and pelvis revealed a large psoas hematoma. Arteriogram revealed lumbar artery bleeding which was treated with embolization. Anticoagulation therapy, while indicated in COVID-19 patients, has its own challenges and guidelines describing dosages and indications in this disease are lacking. Rare bleeding complications such as psoas hematoma should be kept in mind in patients who become hemodynamically unstable, warranting prompt imaging for diagnosis and treatment with arterial embolization, thus eliminating need of surgical intervention.
\end{abstract}

Keywords: Retroperitoneal hemorrhage; COVID-19; Lumbar artery bleeding; Arterial embolization; Enoxaparin

Manuscript submitted June 6, 2020, accepted June 13, 2020

Published online June 25, 2020

aDepartment of Medicine, Jersey Shore University Medical Center, Hackensack Meridian Health, Neptune, NJ, USA

${ }^{b}$ Department of Pulmonology and Critical Care, Jersey Shore University Medical Center, Hackensack Meridian Health, Neptune, NJ, USA

'Department of Interventional Radiology, Jersey Shore University Medical Center, Hackensack Meridian Health, Neptune, NJ, USA

${ }^{\mathrm{d} C}$ Corresponding Author: Steven Douedi, Department of Medicine, Jersey Shore University Medical Center, Hackensack Meridian Health, Neptune, NJ 07753, USA. Email: Steven.Douedi@Hackensackmeridian.org

doi: https://doi.org/10.14740/jocmr4256

\section{Introduction}

Critically ill patients with coronavirus disease 2019 (COVID-19) are at increased risk of developing hypercoagulable state as evidenced clinically by stroke in young patients as well as laboratory investigations like elevated D-dimer levels warranting therapeutic anticoagulation [1]. The pathophysiology behind this elevated coagulation phenomenon has been suspected to be a result of increased pro-inflammatory cytokines such as interleukin-6 (IL-6), IL-1 beta and tumor necrosis factor (TNF) alpha among others which facilitate atherosclerotic changes [2]. To combat this hypercoagulable state, patients infected with COVID-19 should be properly anticoagulated to reduce ischemic risk. Lumbar artery bleeding causing retroperitoneal hemorrhage is usually seen due to trauma, anticoagulation therapy, malignancies, coagulopathies or hemodialysis [3]. To the best of our knowledge, we report first case of retroperitoneal hemorrhage due to lumbar artery bleeding in a patient of COVID-19 being treated with enoxaparin, highlighting importance of judicious use of anticoagulation in COVID-19 patients as well as the need of prompt radiological investigation and treatment of bleeding.

\section{Case Report}

A 69-year-old man with past medical history of coronary artery disease (CAD) on $81 \mathrm{mg}$ of aspirin daily, hypertension and type 2 diabetes mellitus presented with fever, malaise and progressive shortness of breath for a week. Vitals on admission showed temperature of $103.1^{\circ} \mathrm{F}$, pulse of 106 beats per minute, respiratory rate of 18 per minute, blood pressure of $142 / 70 \mathrm{~mm} \mathrm{Hg}$ and saturation of $89 \%$ on room air. Physical examination revealed decreased breath sounds bilaterally at base with crepitations. Laboratory investigation showed hemoglobin $12.7 \mathrm{~g} / \mathrm{dL}$ (normal value: $12.0-17.5 \mathrm{~g} / \mathrm{dL}$ ), white blood cell count of $4.5 \times 10^{3}$ (normal value: $4.5-11.0 \times 10^{3} /$ $\mu \mathrm{L}$ ), platelet count of $164 \times 10^{3}$ (normal value: $140-450 \times$ $10^{3} / \mu \mathrm{L}$ ), neutrophil $76.4 \%$ (normal value: $50.0-70.0 \%$ ), lymphocyte $14.9 \%$ (normal value: $25.0-43.0 \%$ ), absolute lymphocytes 0.7 (normal value: $1.5-3.5 \times 10^{3} / \mu \mathrm{L}$ ), C-reactive protein (CRP) $12.07 \mathrm{mg} / \mathrm{dL}$ (normal value: $0.00-0.74 \mathrm{mg}$ / 


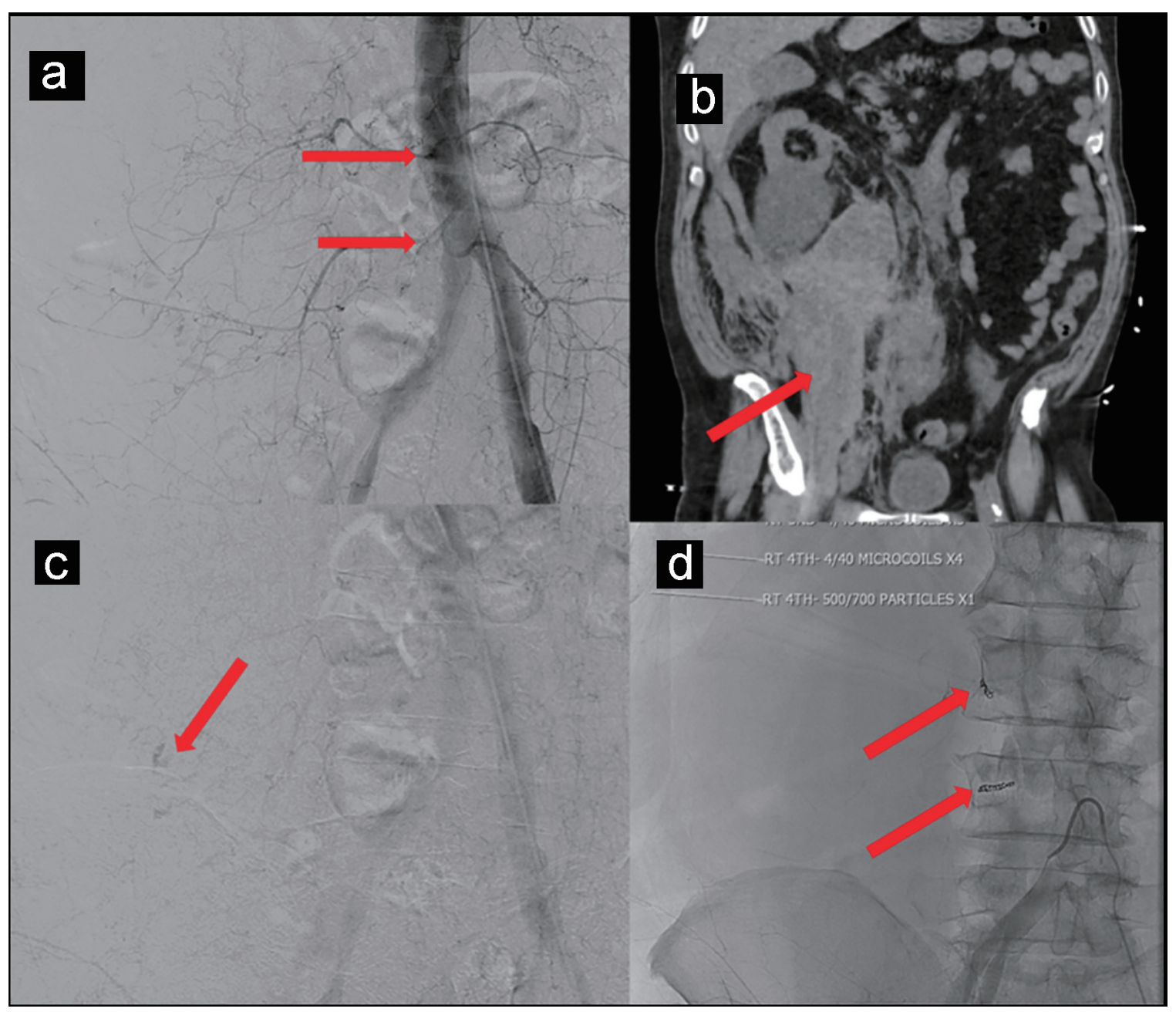

Figure 1. (a, b) CT scan of the abdomen and pelvis without contrast showing hemorrhage along entire length of right psoas muscle measuring $10 \times 17 \times 24 \mathrm{~cm}$. (c) Subtle extravasation identified from branches of the right L3 and L4 lumbar arteries. (d) Four $4 \mathrm{~mm}$ in diameter by $40 \mathrm{~mm}$ in length microcoils placed to control bleeding.

$\mathrm{dL}$ ) and D-dimer $570 \mathrm{ng} / \mathrm{mL}$ (normal value: $<500 \mathrm{ng} / \mathrm{mL}$ ). Influenza A and B serology was negative, COVID-19 RT-PCR was positive, and chest X-ray showed bilateral infiltrates at the lung bases. The patient was started on hydroxychloroquine, azithromycin, supplemental oxygen with nasal cannula and daily $40 \mathrm{mg}$ subcutaneous prophylactic enoxaparin. Over the course of hospitalization, the patient's respiratory condition worsened. Repeat chest X-ray showed worsening of bilateral infiltrates. He was transitioned to optiflow $(100 \%$ $\mathrm{FiO}_{2}$ at $55 \mathrm{~L} / \mathrm{min}$ ). His dimer up trended to $1,763 \mathrm{ng} / \mathrm{mL}$. The patient was continued on aspirin for CAD and was started on weight-based $(1 \mathrm{mg} / \mathrm{kg})$ therapeutic enoxaparin for concern of hypercoagulable state. On day 20 of hospitalization, the patient started complaining of abdominal pain, but his physical examination was unremarkable. Vitals at the time showed pulse of 113 beats per minute, blood pressure $74 / 45 \mathrm{~mm} \mathrm{Hg}$ with a hemoglobin level of $8.9 \mathrm{~g} / \mathrm{dL}$, suggestive of hypovolemic shock. Computed tomography (CT) scan of the abdomen and pelvis without contrast showed hemorrhage along entire length of right psoas muscle measuring $10 \times 17 \times 24$ cm (Fig. 1a, b). The patient was transferred to intensive care unit (ICU) and was resuscitated with $2 \mathrm{~L}$ intravenous fluid, 3 units of packed red blood cells (PRBC) and vasopressors. He did not respond appropriately to fluids and his vasopressor requirement increased with post-transfusion hemoglobin of $6.6 \mathrm{~g} / \mathrm{dL}$. He received two more units of PRBC and 1 unit of fresh frozen plasma. Due to patient's age, clinical condition and current use of anticoagulation arterial embolization was planned instead of surgery. Under ultrasound guidance, left common femoral artery was accessed and abdominal aortogram was performed. Subtle extravasation was identified from branches of the right L3 and L4 lumbar arteries (Fig. 1c). Both arteries were selectively catheterized with a 5 French Mickelson catheter through which a high flow microcatheter was placed in coaxial fashion. Active extravasation was identified along the superior margin of the right iliac bone on the frontal view. One-quarter vial of $500-700 \mu \mathrm{m}$ Embosphere particles were administered followed by four 4 $\mathrm{mm}$ in diameter by $40 \mathrm{~mm}$ in length microcoils (Fig. 1d). After procedure, there was no active contrast extravasation 
identified. The patient received one more unit of PRBC and his hemoglobin stabilized to $7.9 \mathrm{~g} / \mathrm{dL}$. Vasopressor requirement eventually decreased and eventually discontinued. Repeat CT scan showed decreased size of hematoma $8.1 \times$ $11.3 \times 21 \mathrm{~cm}$. The patient remained in ICU due to COVID-19 pneumonia with a stable hemoglobin level and no further bleeding and was eventually weaned off optiflow and transferred to the medical floors for further management.

\section{Discussion}

One of the causes of high mortality in COVID-19 patients is increased risk of disseminated intravascular coagulation (DIC) and venous thromboembolism as evidenced by altered coagulation profile like elevated D-dimers [1]. It has been purposed this hypercoagulable phenomenon is due to increased proinflammatory cytokines, mainly IL-6 in COVID-19-infected patients [2]. These inflammatory cytokines facilitate atherosclerotic changes through local inflammation, plaque rupture and hemodynamic changes [2]. Pro-inflammatory cytokines have also been found to be elevated and one of the causes of DIC which can be seen in septic patients $[4,5]$. This hypercoagulable state causes microvascular thrombi causing multiorgan failure and death and should be treated with antithrombotic agents like heparin [5]. Treatment with therapeutic dose anticoagulation therapy may prove beneficial but still controversial. Anticoagulation treatment and additional anti-platelet medications, such as aspirin as seen in our patient, increase the risk of major bleeding complications like retroperitoneal hemorrhage. Here we report a case of retroperitoneal hemorrhage in a COVID-19 patient on $81 \mathrm{mg}$ aspirin daily for CAD and enoxaparin $40 \mathrm{mg}$ daily due to COVID-19-induced hypercoagulable state ultimately treated with arterial embolization [1].

The psoas muscle can accumulate up to 10 times its own volume and a psoas hematoma due to retroperitoneal hemorrhage can present with hypotension, abdominal pain, or drop in hemoglobin as seen in our patient $[6,7]$. CT scan remains the initial imaging of choice for suspected retroperitoneal hemorrhage. Initial treatment consists of cessation of anticoagulants, transfusion therapy, volume resuscitation and supportive measures [8]. Hemodynamically unstable patients on anticoagulation therapy with unpredictable large soft tissue hematomas can be treated with arterial embolization as it is minimally invasive with quick therapeutic effect when compared with surgical treatment. When active psoas hematoma bleeding is seen, arterial embolization of third and fourth lumbar arteries is usually warranted which remains a technical challenge as it requires selective catheterization of bleeding artery, embolization and post-embolization studies to document successful treatment as seen in our patient [9].

There is an expectation that another wave of COVID-19 will hit our hospital system [10]. In preparation of this expected event, we in scientific community should start to prepare evidence-based guidelines on how to approach this complex disease. We currently know that pulmonary thrombi in COVID-19 develop as a consequence of vascular damage associated with viral infection and severe inflammation as evidenced by their D-dimer levels and inflammatory markers and increase in multiple thrombolytic factors like IL- 6 and antiphospholipid antibodies $[2,11,12]$. Pathophysiological speaking, high-dose heparin in this setting may not only be ineffective, but it may also be harmful, possibly contributing to the described hemorrhagic component of microangiopathy [12]. We definitely need further high-quality studies preferably randomized trials to answer whether full dose heparin is warranted, how long it should be continued after discharge of COVID-19 patients and is it better than only prophylactic dose heparin to decrease allcause mortality. However, we already know that using highdose heparin increases fatal and major bleeding rates, thus this case report and others are raising these concerns as we need better evidence to support this practice $[6,13]$.

\section{Conclusion}

COVID-19 is known to cause an increase in inflammatory markers leading to a hypercoagulable state requiring the use of anticoagulant therapy. While the use of anticoagulation deems appropriate in COVID-19 as per literature and our clinical observations, dosing and guidelines regarding this are lacking and desperately needed. Clinicians should also be vigilant about bleeding complications such as retroperitoneal hemorrhage in COVID-19 patients on anticoagulation. In such instances by donning appropriate personal protective equipment (PPE), arterial embolization can be performed as it remains safe, effective and less invasive with good outcomes when compared with surgery as described in our case report.

\section{Acknowledgments}

None to declare.

\section{Financial Disclosure}

None to declare.

\section{Conflict of Interest}

None to declare.

\section{Informed Consent}

The patient described in the case report had given informed consent for the case report to be published.

\section{Author Contributions}

IP and AA: visualization and writing original draft, review and editing. SD, VU, UM, EC and DF: writing review and editing. All authors have read and agreed to the published version of the manuscript. 


\section{Data Availability}

The authors declare that data supporting the findings of this study are available within the article.

\section{References}

1. Cattaneo M, Bertinato EM, Birocchi S, Brizio C, Malavolta D, Manzoni M, Muscarella G, et al. Pulmonary embolism or pulmonary thrombosis in COVID-19? Is the recommendation to use high-dose heparin for thromboprophylaxis justified? Thromb Haemost. 2020.

2. Zhou F, Yu T, Du R, Fan G, Liu Y, Liu Z, Xiang J, et al. Clinical course and risk factors for mortality of adult inpatients with COVID-19 in Wuhan, China: a retrospective cohort study. Lancet. 2020;395(10229):1054-1062.

3. Marty B, Sanchez LA, Wain RA, Ohki T, Marin ML, Bakal C, Veith FJ. Endovascular treatment of a ruptured lumbar artery aneurysm: case report and review of the literature. Ann Vasc Surg. 1998;12(4):379-383.

4. Wada H, Tanigawa M, Wakita Y, Nakase T, Minamikawa $\mathrm{K}$, Kaneko T, Ohiwa M, et al. Increased plasma level of interleukin-6 in disseminated intravascular coagulation. Blood Coagul Fibrinolysis. 1993;4(4):583-590.

5. Papageorgiou C, Jourdi G, Adjambri E, Walborn A, Patel P, Fareed J, Elalamy I, et al. Disseminated intravascular coagulation: an update on pathogenesis, diagnosis, and therapeutic strategies. Clin Appl Thromb Hemost. 2018;24(9_suppl):8S-28S.

6. Surani S, Estement B, Manchandan S, Sudhakaran S,
Varon J. Spontaneous extraperitoneal lumbar artery hemorrhage. J Emerg Med. 2011;40(6):e111-114.

7. Qanadli SD, El Hajjam M, Mignon F, Bruckert F, Chagnon S, Lacombe P. Life-threatening spontaneous psoas haematoma treated by transcatheter arterial embolization. Eur Radiol. 1999;9(6):1231-1234.

8. Hamid RS, ul HT, Chishti I, Azeemuddin M. Post traumatic avulsion of lumbar artery: a rare cause of retroperitoneal haemorrhage treated by glue embolization. J Pak Med Assoc. 2010;60(6):487-489.

9. Amole AO, Kathuria MK, Ozkan OS, Gill AS, Ozkan EO. Lumbar artery laceration with retroperitoneal hematoma after placement of a G-2 inferior vena cava filter. Cardiovasc Intervent Radiol. 2008;31(6):1257-1259.

10. Xu S, Li Y. Beware of the second wave of COVID-19. Lancet. 2020;395(10233):1321-1322.

11. Klok FA, Kruip M, van der Meer NJM, Arbous MS, Gommers D, Kant KM, Kaptein FHJ, et al. Confirmation of the high cumulative incidence of thrombotic complications in critically ill ICU patients with COVID-19: An updated analysis. Thromb Res. 2020;191:148-150.

12. Bikdeli B, Madhavan MV, Jimenez D, Chuich T, Dreyfus I, Driggin E, Nigoghossian C, et al. COVID-19 and thrombotic or thromboembolic disease: implications for prevention, antithrombotic therapy, and followup: JACC State-of-the-Art review. J Am Coll Cardiol. 2020;75(23):2950-2973.

13. Janik V, Martinek V, Padr R, Lisy J, Neuwirth J, Pafcugova J, Vanecek T, et al. Embolization of lumbar artery due to retroperitonal bleeding following renal biopsy. Nephrol Dial Transplant. 2005;20(4):820-822. 